\title{
Physiological performance of field-released insects
}

\author{
John S. Terblanche* \\ Centre for Invasion Biology, Department of Conservation Ecology and Entomology, Stellenbosch \\ University, Stellenbosch, South Africa \\ *Corresponding author. Email: jst@sun.ac.za; Tel:+27-21-808-9225; Fax:+27-21-808-3304
}

\begin{abstract}
Predicting insect field performance has direct value for control programs seeking increased efficacy while simultaneously providing insights into field physiology and responses to environmental variability. Recent studies of field-released insects have made significant progress in three main areas. First, the trade-offs associated with thermal history relative to abiotic conditions on a given day have been repeatedly demonstrated in several taxa. Cold-acclimated insects released into hotter environments typically suffer performance costs - but do better than controls - in cooler environments suggesting both costs and benefits to physiological adjustments. Second, molecular mechanisms explored to date suggest complex underlying associations with recapture rates. Third, there has been significant progress in strengthening the link between traits scored in the laboratory as indicators of field performance. The overarching conclusion from this developing field suggests that physiological adjustments can make large, and in at least several cases, predictable changes in performance under field conditions. Further research is likely to contribute important insights into variation in field performance of insects.
\end{abstract}

Key-words: biological control; flexibility; beneficial acclimation; developmental plasticity; oxidative damage; pest control 
Terblanche - field physiological performance

\section{Introduction}

Understanding organismal responses to environmental variability is critical to predicting and managing population and species responses at a range of spatial and temporal scales. Knowledge of dispersal patterns of organisms, for example, feeds directly into understanding the structure of a species' geographic range, metapopulation dynamics, colonisation and invasion potential [1] with direct implications for predicting the impacts of environmental variability at various temporal scales (e.g. climate change, weather fronts). Similarly, a wide array of applied pest or vector management strategies requires some information on movement in the landscape [2]. It is clear however, that dispersal rates - and physiological performance more generally - are intrinsically linked to current and past environmental conditions. For example, flight ability, walking speed and jumping distance of insects typically increase up to an optimum temperature, thereafter declining rapidly [e.g., 3-6]. Population abundance can therefore be viewed as the outcome of several abiotic and biotic variables and their interactions with organism phenotypes of performance and stress resistance. Since insects have likely evolved to perform optimally within their habitat, they can have relatively narrow ranges of abiotic conditions for optimal performance. Outside of this range, insects must withstand or avoid stress, performance and fitness can be reduced, and the risk of mortality increases. Numerous studies demonstrate the sensitivity and behavioural responses of insects to microclimatic variation in the field (e.g., [7]). The temperature-dependence of various biological and reaction rates is also widely acknowledged and extensively documented $[8,9]$, although the influence of prior conditions on such rates, either within or among generations, is less obvious and in some cases even highly contentious (discussed in [10]). Typically, laboratory estimates, obtained under controlled conditions, are used to make approximations and inferences regarding field physiology and performance, although the validity of such an approach is increasingly questioned (e.g., [11]).

Despite ongoing debate surrounding the nature and form of trait plasticity and its potential benefits for field performance and evolutionary fitness, it is increasingly well appreciated that relatively small changes in thermal conditions during laboratory culture, or perhaps even increased temperature variability (e.g. $[12,13])$, can result in increased performance when exposed to similar conditions at a later stage in the field or semi-field environment (e.g. [14]). For example, cold rearing of the ladybird biocontrol agent, Adalia bipunctata, results in a significant increase in its predation rate on aphids at cooler temperatures ([11], Fig. 1B). Such predictable variation in performance, while providing valuable tests of evolutionary theory of plastic trait responses (e.g. beneficial acclimation and alternative hypotheses; [10]), can also be a useful tool for enhancing pest management practises, especially given the wide range of conditions under which laboratory- or mass-reared insects are expected to perform for biological control and sterile-mating control methods to succeed [11].

Area-wide integrated pest management strategies typically seek to release laboratory- or massreared insects into field environments to elicit population control either through reproduction and inherited sterility between wild and genetically modified individuals of the same species or some form of mating disruption (reviewed in [15]). While the fact that small changes in environmental or rearing conditions can result in significant improvements in field performance for such approaches has not been lost in the applied pest management field (e.g. [16]) these concepts are not widely utilised. This is especially significant since large numbers of individuals must be released to achieve 
measurable levels of population control, and that financial expenditure is typically high in pest management or vector control programs, especially when carried out on an area-wide basis (ranging from a few cents to US \$1-10 per individual depending on the program, location, operational scale and focal species). Consequently, operational costs become critical to program continuity when costs are shared among partners or users (e.g. farmers), and can determine the continuation or initial investment of state-funded programs $[15,17]$. To date, the trend has largely been to increase factory output (i.e. numbers) in mass-release of control agents, rather than to develop a more costeffective, physiologically-optimised insect for release (reviewed in [11]). Here, I therefore aim to review recent progress on understanding drivers of field performance of insects under three main themes. First, I shall discuss thermal acclimation and its relative costs and benefits, then discuss studies which have sought to identify underlying mechanisms associated with variation in field performance, and finally, the importance of linking field and laboratory performance of insects in a predictable, accurate manner. Together, these three themes perhaps constitute the boundary of current knowledge, and thus probably represent major limitations in understanding field performance of insects for applied or basic biology.

\section{Costs and benefits to physiological adjustments}

Physiological adjustments to changes in environmental conditions are widely demonstrated for insects and are expected to provide performance advantages under field conditions (e.g. at transitions between seasons or weather fronts). Acclimation can be described as the physiological response to altered conditions. A classic example is the dramatically increased heat tolerance (i.e. lower mortality) induced if Drosophila are given a mild (non-lethal) heat shock prior to what would have otherwise been a lethal heat exposure (e.g. [18]). The effects of changing environmental conditions can have a broad influence on a diverse range of traits from morphology and life-history to stress tolerance and physical performance. Further complexity in understanding physiological responses to changing conditions occurs because dissimilar responses may be elicited over a range of time-scales (minutes, hours, days, generations), and similar responses can be induced by a range of stressors (acute, chronic). The differences among these potentially distinct forms of phenotypic plasticity are typically not well established outside model species (e.g., [19]; reviewed by [20]). Broadly, 'hardening' typically refers to acute responses (e.g. cold or heat shock), while 'acclimation' encompasses the physiological responses to more chronic, sub-lethal conditions in the laboratory (acclimatization is the field equicalent of acclimation). Moreover, some traits respond more than others to an equivalent treatment. For example, thermal acclimation often induces a greater acclimation response in low temperature tolerance than high temperature tolerance traits (e.g. [21, 4]), which is also of interest in forecasting climate change-related responses given concerns for managing pest species in future warming scenarios, unpredictable or highly variable environments (e.g., [22]). In most cases, tests of acclimation responses have focused on the beneficial acclimation hypothesis, in which it is expected that acclimation to a particular environment gives a performance advantage in that environment relative to an individual not given the opportunity to acclimate [10], although little consensus on plasticity responses has emerged thus far. Such acclimation responses are more frequently explored under controlled laboratory conditions (e.g. [23]; reviewed by [24]) and, to a lesser extent, in the field (e.g., [25, 26]; Table 1).

Under field conditions, and especially across a wide range of test conditions (broad thermal range), support is generally found for an advantage to acclimation scored for a range of taxa and study 
systems using diverse performance- or fitness-related traits (Table 1). Recently, Chidawanyika and Terblanche [21] aimed to determine if field performance of pest insects in a sterile insect release program could be influenced by thermal acclimation by subjecting developing larvae to temperatures both above and below optimal rearing conditions for a week. Codling moths (Cydia pomonella) reared for a week at cooler temperatures were recaptured significantly more ( $\sim$ two-fold) than control or warm-acclimated moths under field conditions that were cooler (lower ambient temperature). By contrast, under warm field conditions, cold-acclimated moths were recaptured significantly less than control moths and far less than warm-acclimated moths (Fig. 1A).The performance advantages of rearing or short-term exposure at a temperature matching the environment experienced later on thus come at a cost; improved physiological or behavioural performance in one environment comes at the expense of performing far worse under the opposite conditions to those used for acclimation (or in environments opposite to those experienced previously) or with a lifetime fitness cost (e.g. reduced longevity or fecundity) (e.g., [25, 27]). There are, however, some examples of studies performed under field conditions which have documented little or no obvious costs (e.g. no change in parasitism rates in the laboratory or under mild field conditions, [26]), but these have been typically explored across a narrower range of thermal conditions in the field and it is therefore unclear if elevated high temperature performance gained from warm acclimation would have simultaneously resulted in a performance reduction under colder test conditions. Thus, different kinds of performance or fitness 'costs' can be expected and further studies should carefully define fitness costs from performance costs in a particular environment.

Previous work on Drosophila found a similar 'cost and benefits'-type result in the field, such that cold-acclimated flies performed better (scored as recapture rate at food bait stations) than control flies, and fared far worse in hot conditions. However, one notable difference between the Kristensen et al. [25] and Chidawanyika and Terblanche [21] studies is that, in the case of Drosophila, the responses of laboratory-scored traits of heat and cold resistance did not match the performance responses scored in the field trials for their relative acclimation effects, suggesting that the link between laboratory and field performance is subtle and perhaps more complex than generally appreciated. Therefore, the rather striking and seemingly intuitive results from the field remain at odds with some laboratory studies and perhaps weaken any future conclusions reached from studies that only consider the laboratory environment [28]. Indeed, if such a result is found more broadly it calls into question the utility of laboratory studies for predicting field biology (see also recent discussions by $[29,30])$.

\section{Molecular mechanisms and field tests of performance}

Although the mechanisms of physiological responses to temperature are well-explored in insects in the laboratory (e.g., [31, 32, 12]), understanding how and why release-recapture results have provided such strong patterns of costs and benefits is essentially unclear from a mechanistic viewpoint $[28,33]$. Without a mechanistic understanding of the variation in field performance, it can be argued that these results are largely correlative. Several earlier studies have attempted to link a particular biochemical response (for example, variation in energy metabolism), or known stress response pathway (such as heat shock protein), with field performance (e.g., [34, 35]). Recently, Sørensen et al. [36] showed that $\mathrm{Hsf}^{+}$mutant flies, which could mount a heat hardening response, tended to have better field performance (recapture rates) than null flies unable to mount a heat 
response ( $\mathrm{Hs} \mathrm{f}^{0}$ flies) although both mutant lines performed poorly relative to wild type flies even under warm field conditions. The poor performance of the mutant lines was not apparent under laboratory trial conditions when scored as a variety of relatively standard stress resistance traits (e.g. desiccation resistance, chill coma recovery, heat resistance). Roberts et al. [37] also attempted to link heat shock protein 70 ( $h s p 70$ ) gene copy number with flight and walking performance in the laboratory. In their study the role of copy number was more clearly linked to locomotor, but not flight, performance. Both these studies suggest a limited role of the heat shock response for field performance - even under field conditions where flies are known to experience heat stress resulting in elevated hsp70 expression [38], hsp70 copy number variants result in developmental abnormalities [39], and in which novel heat waves can result in whole-genome changes [40]. Thus, there are likely to be highly trait-specific associations with key stress pathways or genes. Recent studies have also sought to associate dispersal-prone individual's metabolomic and transcriptomic profiles with those remaining stationary upon release in the field (e.g., [28, 33]) to gain insight into field physiological responses. Briefly, these studies have showed that although metabolites may shifted in distinct ways among flies from different thermal regimes, these are not necessarily easily or directly coupled to observed field responses in a predictable manner. These latter results are of particular interest if we aim to move towards the accurate prediction of field performance, if we seek to genetically modify a given stress pathway to alter field performance, or if there is a need to transition from a given laboratory-scored trait to predict field efficacy upon release.

In a recent novel study, Lopez-Martinez et al. [41] took a different approach and instead sought to assess the role of oxygen deprivation (anoxia), and the antioxidant stress pathway, for its role in flight performance of Cactoblastis cactorum moths in laboratory trials. Here, the authors demonstrated several valuable performance improvements in moths that were treated with anoxia prior to or during gamma irradiation, including elevated flight performance and maintenance of key mating behaviors. Trials examining if these results apply in the field are presently underway (G. Lopez-Martinez, pers. comm.). Similarly pronounced effects of thermal history have been shown for ladybird biocontrol agent performance against aphids in mesocosm tests [14] and for flight performance of oriental fruit moths tested in the laboratory on flight mills [23]. To summarize, molecular mechanisms explored to date suggest complex underlying associations with field traits of performance (e.g. recapture rates), yet this remains a critical area of research in moving towards a predictive framework of field physiology.

\section{Linking laboratory and field estimates of performance}

There is a long history of research seeking to understand dispersal phenotypes and performance under field conditions (e.g., [42-44]), typically by morphological association (e.g., [43]). The latter is of interest for several broad reasons (climate change, habitat fragmentation, invasion biology), but perhaps most significantly for applied pest management is the ability to use a selected, laboratoryscored phenotype as a reflection of field performance, and indeed, to predict accurately the field performance of laboratory- or mass-reared insects upon release. More recently, work has begun documenting remarkably strong effects of even a single nucleotide polymorphism in a selected key metabolic pathway enzyme (e.g. phosphoglucose isomerase, Pgi) that can be associated with elevated field performance under certain environmental conditions (e.g., [44]). For example, in the butterfly Melitea cinxia heterozygous individuals at a single nucleotide polymorphism in the Pgi gene dispersed further and at lower temperatures than homozygous individuals [44]. Given the 
contrasting outcomes of recent field studies, in which either laboratory acclimation responses match field physiological responses or not depending on the choice of assay and traits considered, (c.f. Kristensen et al. [25] with Chidawanyika and Terblanche [21]), it is critical that further studies examine field performance in the three major operational environments (laboratory, semi-field, and field). This will allow better understanding of when and where a given trait's association with field performance breaks down. Furthermore, obtaining a better mechanistic understanding of variation in field performance is a broader goal that likely of interest to other research fields (such as dispersal, metapopulation dynamics and invasion biology). Despite this growing body of work examining field dispersal and underlying mechanisms, few studies have explicitly documented clear biochemical mechanisms associated with both laboratory and field performance (but see e.g., [34]). The value of furthering knowledge in this area is that projects can begin to focus on increased efficacy, and ultimately in developing well-tailored, highly task-specific, and physiologicallyoptimised pest-control methods. As mentioned earlier, this in turn could lead to a more costeffective, rather than an inundated, approach to releasing insects in population control methods than is presently the case [11].

\section{Conclusion}

Several conclusions can be reached from this non-exhaustive review of field performance, of which three are perhaps most significant. First, the trade-offs associated with thermal history relative to thermal conditions on a given day have been repeatedly demonstrated in the field. Cold-acclimated insects released into hotter environments typically suffer performance costs but do better than controls in cooler environments suggesting both costs and benefits to physiological adjustments. Second, molecular mechanisms explored to date suggest complex underlying associations between heat shock protein 70 responses or copy number, or pathways of energy metabolism (e.g. enzyme isoforms), and recapture rates. Third, there has been significant progress in strengthening the link between traits scored in the laboratory as indicators of field performance. The overarching conclusion from this developing field suggests that physiological adjustments can make large, and in several cases, predictable changes in performance under field conditions. Understanding these responses under laboratory and field conditions is critical to inform knowledge of field physiological performance of insects. While additional field studies would be useful for further understanding of specific mechanisms or stress responses and their relative importance under field conditions, the studies to date suggest that establishing underlying mechanisms for performance variation under field circumstances will be challenging and likely involve multiple changes at various hierarchical levels within the organism.

What makes understanding field physiological performance especially challenging is if a wide range of abiotic or biotic factors are to be accounted for in their potential influence on field performance. This perhaps suggests that one should expect laboratory trials to fail to capture field performance of physiological responses accurately, but if this is the case, the question then becomes by how much and to what degree this is an acceptable compromise between effort expended and (reliable) information gained? It is increasingly clear that even small variations in the method employed to score laboratory traits, particularly in tolerance or stress resistance assays, can differ in fundamental and subtle ways (reviewed in e.g., [29]) and this is likely to also be the case in performance assays. It is however possible to capture elements of variation in field performance in relatively simple laboratory assays (see e.g., [41]) which can significantly enhance the prediction ability of field 
responses, which therefore helps achieving the applied knowledge that may be of primary interest. A key factor is knowing when and where results of laboratory assays apply for a given system in the field and if any generalities across diverse systems may emerge in the future. This suggests broadening the net, especially in terms of focal species examined and methods of scoring field and laboratory performance. It would also be useful, especially from an applied perspective, to understand the relative costs and benefits of various forms of physiological responses at a range of time scales. Few studies, if any, have explicitly sought to dissect longer-term acclimation responses from shorter, more acute responses (e.g. hardening) under field conditions. Given that rearing for a longer period is going to be more time-consuming, and therefore perhaps less appealing from a financial or logistic perspective in mass-rearing facilities, it would be of value to know if hardening responses can be substituted for the more long-term acclimation traditionally employed in several studies to date. Furthermore, demonstration of mechanistic effects in all three standard operational environments typically employed, from laboratory, semi-field (e.g. cages or greenhouses) and in the field, is especially valuable since it allows the determination of when and where known effects disappear, if at all. Such multi-faceted approaches on non-model organisms are likely to greatly enhance linking information on field performance to laboratory assays and vice versa, and in establishing any generalities.

\section{Acknowledgements}

JST is supported by the National Research Foundation incentive funding scheme (IFR) and HortGro Science. Thanks to Ray Huey for continued inspiration and Brent Sinclair, Torsten Kristensen and Jesper Sørensen for several productive discussions and constructive comments on this review.

\section{References}

[1] Clobert J, Baguette M, Benton TG, Bullock JM: Dispersal Ecology and Evolution. Oxford University Press; 2012.

[2] Mazzi D, Dorn S: Movement of insect pests in agricultural landscapes. Ann Appl Biol 2012, 160:97-113.

[3] Alford L, Hughes GE, Blackburn TM, Bale JS: Walking speed adaptation ability of Myzus persicae to different temperature conditions. Bull Ent Res 2012, 102:303-313.

[4] Lachenicht MW, Clusella-Trullas S, Boardman L, le Roux C, Terblanche JS: Effects of acclimation temperature on thermal tolerance, locomotion performance and respiratory metabolism in Acheta domesticus L. (Orthoptera: Gryllidae). J Insect Physiol 2010, 56:822-830.

[5] Lehmann F-O: Ambient temperature affects free-flight performance in the fruit fly Drosophila melanogaster. J Comp Physiol B 1999, 169:165-171.

[6] Snelling EP, Becker CL, Seymour RS: The effects of temperature and body mass on jump performance of the locust Locusta migratoria. PLoS One 2013, 8:e72471.

[7] Johns et al. 2012

[8] Dell Al, Pawar S, Savage VM: Systematic variation in the temperature dependence of physiological and ecological traits. Proc Natl Acad Sci USA 2011, 108:10591-10596.

[9] Irlich UM, Terblanche JS, Blackburn TM, Chown SL: Insect rate-temperature relationships: environmental variation and the metabolic theory of ecology. Am Nat 2009, 174:819-835. [10] Wilson RS, Franklin CE: Testing the beneficial acclimation hypothesis. Trends Ecol Evol 2002, 17:66-70. 
[11] Sørensen JG, Addison MF, Terblanche JS: Mass-rearing of insects for pest management: Challenges, synergies and advances from evolutionary physiology. Crop Prot 2013, 38:87-94. [12] Boardman L, Sørensen JG, Terblanche JS: Physiological responses to fluctuating thermal and hydration regimes in the chill susceptible insect, Thaumatotibia leucotreta. J Insect Physiol 2013, 59:781-794.

[13] Lalouette L, Williams CM, Hervant F, Sinclair BJ, Renault D: Metabolic rate and oxidative stress in insects exposed to low temperature thermal fluctuations. Comp Biochem Physiol A 2011, 158:229-234.

[14] ** Sørensen CH, Toft S, Kristensen TN: Cold-acclimation increases the predatory efficiency of the aphidophagous coccinellid Adalia bipunctata. Biol Contr 2013, 65:87-94.

First demonstration of costs and benefits of thermal acclimation in a biocontrol agent. Critical for field applications and understanding field responses to variable conditions.

[15] Dyck VA, Hendrichs J, Robinson AS (Eds): Sterile Insect Technique. Principles and Practise in Area-Wide Pest Management. Springer; 2005.

[16] Chambers DL: Quality control in mass rearing. Ann Rev Ent 1977, 22:289-308.

[17] Enserink M: Welcome to Ethiopia's fly factory. Science 2007, 317:310-313.

[18] Levins R: Thermal acclimation and heat resistance in Drosophila species. Am Nat 1969, 103:483-499.

[19] *Colinet H, Hoffmann AA: Comparing phenotypic effects and molecular correlates of developmental, gradual and rapid cold acclimation responses in Drosophila melanogaster. Funct Ecol 2012, 26:84-93.

An important recent demonstration of the distinct molecular responses associated with different forms of thermal responses at different time-scales and under specific acute and chronic conditions.

[20] Chown SL, Terblanche JS: Physiological diversity in insects: ecological and evolutionary contexts. Adv Insect Physiol 2007, 33:50-152.

[21] Chidawanyika F, Terblanche JS: Costs and benefits of thermal acclimation for codling moth, Cydia pomonella (Lepidoptera: Tortricidae): implications for pest control and the sterile insect release programme. Evol App/ 2011, 4: 534-544.

[22] Bebber DP, Ramotowski MAT, Gurr SJ: Crop pests and pathogens move polewards in a warming world. Nature Clim Change 2013, 3:985-988.

[23] *Ferrer A, Mazzi D, Dorn S: Stay cool, travel far: cold-acclimated oriental fruit moth females have enhanced flight performance but lay fewer eggs. Ent Exp Appl 2014, doi:10.1111/eea.12163. A recent example of the pervasive effects of rearing temperature on flight performance under controlled laboratory conditions.

[24] Huey RB, Berrigan DA: Testing evolutionary hypotheses of acclimation. In Animals and Temperature: Phenotypic and Evolutionary Adaptation. Edited by Johnston IA, Bennett AF Cambridge University Press; 1996: 205-237.

[25] Kristensen TN, Hoffmann AA, Overgaard J, Sørensen JG, Hallas R, Loeschcke V: Costs and benefits of cold acclimation in field-released Drosophila. Proc Natl Acad Sci USA 2008, 105:216-221. [26] Thomson LJ, Robinson M, Hoffmann AA: Field and laboratory evidence for acclimation without costs in an egg parasitoid. Funct Ecol 2001, 15:217-221.

[27] Scott M, Berrigan D, Hoffmann AA: Costs and benefits of acclimation to elevated temperature in Trichogramma carverae. Ent Exp Appl 1997, 85:211-219.

[28] Overgaard J, Sorensen JG, Jensen LT, Loeschcke V, Kristensen TN: Field tests reveal genetic variation for performance at low temperatures in Drosophila melanogaster. Funct Ecol 2010, 24:186-195.

[29] Terblanche JS et al.: Ecologically relevant measures of tolerance to potentially lethal temperatures. J Exp Biol 2011, 214:3713-3725. 
[30] Kostal V, Miklas B, Dolezal P, Rozsypal J, Zahradnickova H: Physiology of cold tolerance in the bark beetle, Pitygones chalcographys and its overwintering in spruce stands. J Insect Physiol 2014, 63:62-70.

[31] MacMillan HA, Williams CM, Staples JF, Sinclair BJ: Reestablishment of ion homeostasis during chill coma recovery in the cricket Gryllus pennsylvanicus. Proc Natl Acad Sci USA 2012, 109:2075020755.

[32] *Waagner D, Holmstrup M, Bayley M, Sørensen JG: Induced cold-tolerance mechanisms depend on duration of acclimation in the chill-sensitive Folsomia candida (Collembola). J Exp Biol 2013, 216:1991-2000.

A valuable demonstration of differences in mechanisms of cold tolerance depending on the duration of thermal treatment in a terrestrial arthropod. Critical for understanding field physiology.

[33] *Kristensen TN, Overgaard J, Hoffmann AA, Nielsen NC, Malmendal A: Inconsistent effects of developmental temperature acclimation on low-temperature performance and metabolism in Drosophila melanogaster. Evol Ecol Res 2012, 14:821-837.

Important recent assessment of metabolomic responses of insects acclimated to low temperatures. A key attempt to link variation in field performance with biochemical responses. [34] Dahlhoff EP, Rank NE: Functional and physiological consequences of genetic variation at phosphoglucose isomerase: Heat shock protein expression is related to enzyme genotype in a montane beetle. Proc Natl Acad Sci USA 2000, 97:10056-10061.

[35] Rank NE, Bruce DA, McMillan DM, Barclay C, Dahlhoff EP: Phosphoglucose isomerase genotype affects running speed and heat shock protein expression after exposure to extreme temperatures in a montane willow beetle. J Exp Biol 2007, 210:750-764.

[36] Sørensen JG, Loeschcke V, Kristensen TN: Lessons from the use of genetically modified Drosophila melanogaster in ecological studies: Hsf mutant lines show highly trait-specific performance in field and laboratory thermal assays. Funct Ecol 2009, 23:240-247.

[37] Roberts SP, Marden JH, Feder ME: Dropping like flies: environmentally induced impairment and protection of locomotor performance in adult Drosophila melanogaster. Physiol Bichem Zool 2003, 76: 615-621.

[38] Feder ME, Roberts SP, Bordelon AC: Molecular thermal telemetry of free-ranging adult Drosophila melanogaster. Oecologia 2000, 123:460-465.

[39] Roberts SP, Feder ME: Natural hyperthermia and expression of the heat shock protein Hsp70 affect developmental abnormalities in Drosophila melanogaster. Oecologia 1999, 121:323-329. [40] Rodriguez-Trelles F, Tarrio R, Santos M: Genome-wide evolutionary response to a heat wave in Drosophila. Biol Lett 2013, 9:20130228.

[41] **Lopez-Martinez G, Carpenter JE, Hight SD, Hahn DA: Low oxygen atmospheric treatment improves the performance of irradiation-sterilized male cactus moths used in SIT. J Econ Ent 2014, 107: 185-197.

First demonstration of importance of antioxidant responses in insect performance. [42] Watt WB: Adaptation at specific loci. I. Natural selection on phosphoglucose isomerase of Colias butterflies - biochemical and population aspects. Genetics 1977, 87:177-194.

[43] Kingsolver JG: Experimental analyses of wing size, flight, and survival in the Western White Butterfly. Evolution 1999, 53:1479-1490.

[44] Niitepold K, et al.: Flight metabolic rate and Pgi genotype influence butterfly dispersal rate in the field. Ecology 2009, 90:2223-2232. 
Table 1. A selection of key recent examples of field tests of physiological performance in insects thermally acclimated prior to release and scored for various measures of field performance. In parentheses after each species is the possible application and value of the species for understanding field performance.

\begin{tabular}{|c|c|c|c|}
\hline Trait/parameter & Species & Main result & Reference \\
\hline $\begin{array}{l}\text { Dispersal; } \\
\text { Recapture at } \\
\text { bait stations }\end{array}$ & $\begin{array}{l}\text { Drosophila melanogaster } \\
\text { (Model organism) }\end{array}$ & $\begin{array}{l}\text { - Increased recapture rates of cold-acclimated flies under cold } \\
\text { - } \text { - Worsitions } \\
\text { - Lab trials did not show these expected changes in field performance } \\
\text { - Costs and benefits depending on environmental conditions } \\
\text { - Lab-scored traits reached a different conclusion }\end{array}$ & [25] \\
\hline Parasitism & $\begin{array}{l}\text { Trichogramma carverae } \\
\text { (egg parasitoid/biocontrol } \\
\text { agent) }\end{array}$ & $\begin{array}{l}\text { - } \begin{array}{l}\text { Benefits of heat treatment under warm field conditions } \\
\text { - }\end{array} \text { Field and lab trials in general agreement } \\
\text { - No major costs shown, but a narrower range of conditions tested in } \\
\text { the field }\end{array}$ & [26] \\
\hline $\begin{array}{l}\text { Dispersal; } \\
\text { Recapture at } \\
\text { sex pheromone } \\
\text { traps }\end{array}$ & $\begin{array}{l}\text { Cydia pomonella } \\
\text { (major crop pest/sterile } \\
\text { insect control } \\
\text { programme) }\end{array}$ & $\begin{array}{l}\text { - Cold-acclimated moths recaptured more than controls, or hot- } \\
\text { - Warclimated moths, under cold conditions } \\
\text { - } \text { control moths under warm conditions } \\
\text { - Lab and field trials in agreement } \\
\text { - Strong support for beneficial acclimation hypothesis }\end{array}$ & [21] \\
\hline
\end{tabular}




\section{Figure 1}
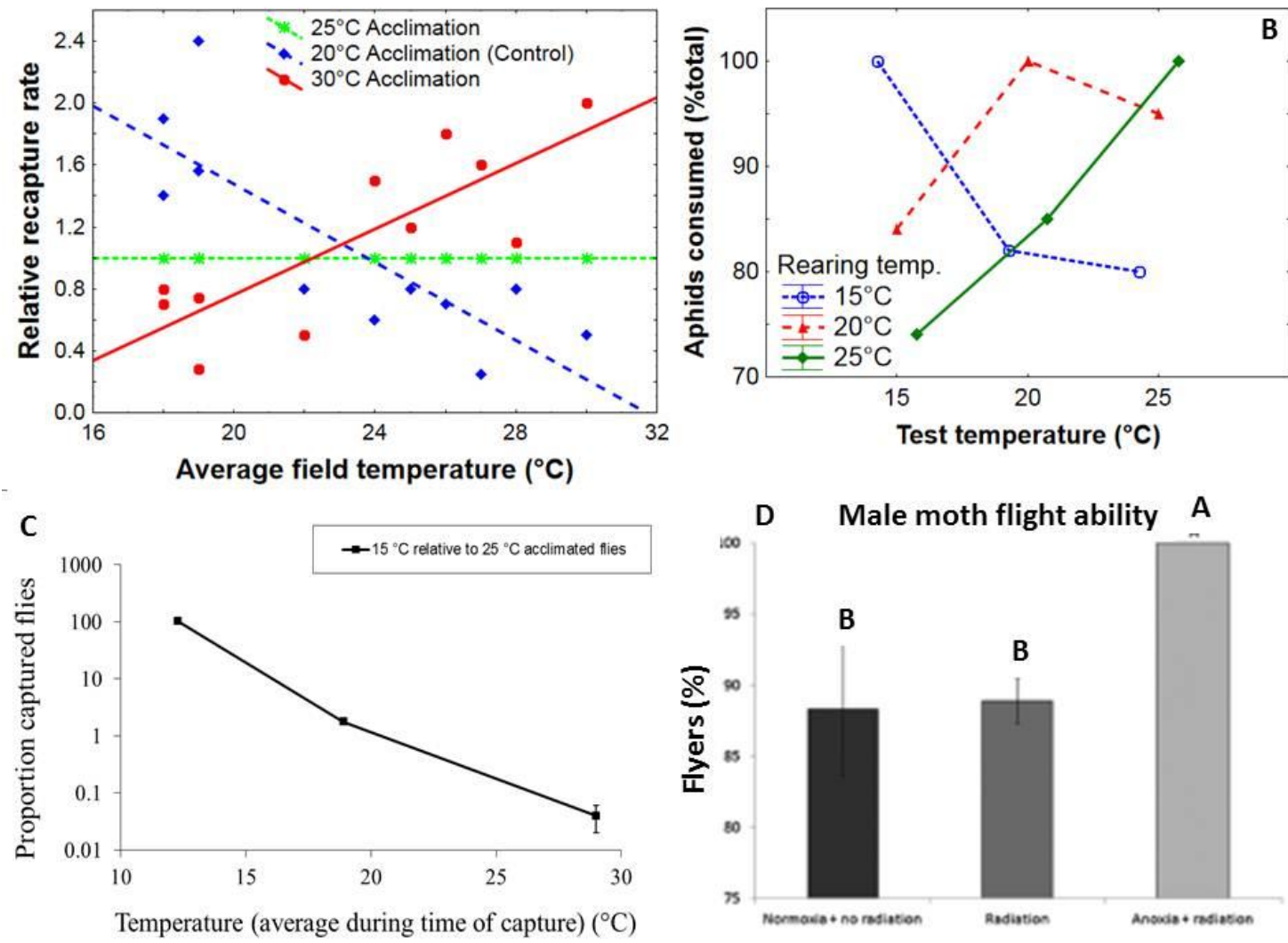

1A) Summary results highlighting the relative change in trap capture of codling moth when acclimated to a particular temperature prior to release across a range of field temperatures (from [21]). B) Summary of relative variation in aphid consumption by the biocontrol agent Adalia bipunctata across a range of test temperatures after acclimation to three thermal environments (from [14]). C) Summary of results showing the relative increase and decrease from control flies in the proportion Drosophila melanogaster recaptured if given a cold acclimation prior to release into a range of thermal environments (from [25]). D) Summary of increase in the number of Cactoblastis moths (\%) willing to fly after treatment with anoxia (no oxygen) + radiation, relative to moths only radiated or normoxia (normal atmospheric oxygen) + no radiation (from [41]). Different letters above groups in panel $D$ indicate statistically different groups. 

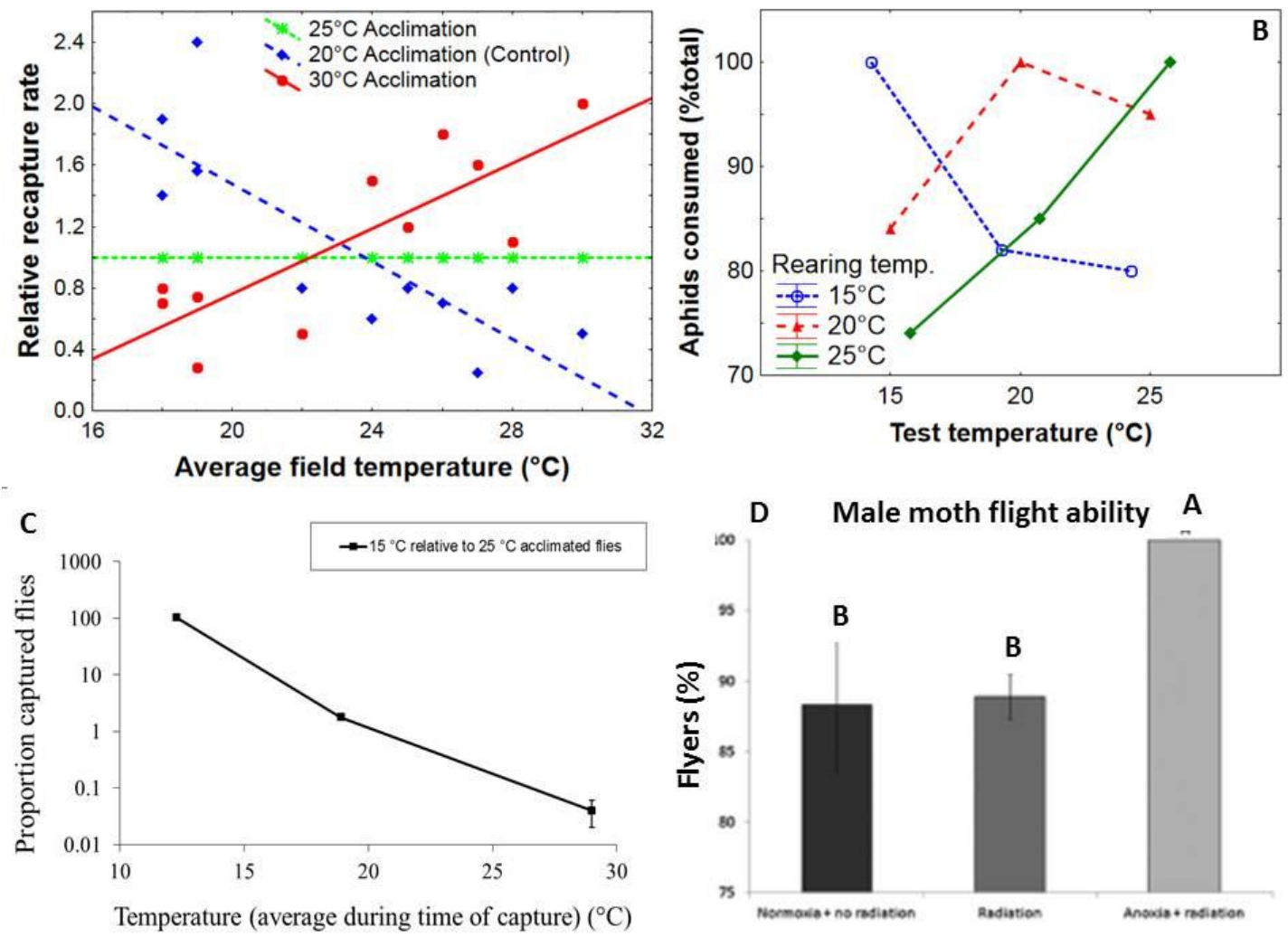